\title{
Influence of cellulose fibers on structure and properties of fiber reinforced foam concrete
}

\author{
Valeriy Fedorov ${ }^{1, *}$, and Aleksey Mestnikov ${ }^{1}$ \\ ${ }^{1}$ Ammosov North-Eastern Federal University, 677000 Yakutsk, Russia
}

\begin{abstract}
One of the promising means of foamed concrete quality improvement is micro-reinforcement by adding synthetic and mineral fibers to the base mix. This research is the first to investigate peculiarities of using recycled cellulose fiber extracted from waste paper for obtaining fiber reinforced foam concrete. The paper presents results of experimental research on the influence of cellulose fibers on structure and properties of fiber reinforced foam concrete by using methods of chemical analysis and scanning electron microscopy. The research determines peculiarities of new formations appearance and densification of binder hydration products in the contact zone between fiber and cement matrix, which boost mechanical strength of fiber reinforced foam concrete. Physico-mechanical properties of fiber reinforced foam concrete were defined depending on the amount of recycled cellulose fiber added to the base mix. It was found that the use of recycled cellulose fibers allows obtaining structural thermal insulating fiber reinforced foam concretes of non-autoclaved hardening of brand D600 with regard to mean density with the following improved properties: compressive strength increased by $35 \%$ compared to basic samples, higher stability of foamed concrete mix and decreased shrinkage deformation.
\end{abstract}

\section{Introduction}

The use of structural and thermal insulating wall materials allows producing lightweight envelope structures that meet modern requirements to thermal protection, environmental and fire safety. Among such materials are cellular concretes, particularly foamed concretes based on binders of hydraulic hardening [1].

In spite of obvious advantages cellular concretes, particularly foamed concrete, has a number of disadvantages which can be observed at all stages of production process. Here belong difficulties of producing uniform foam, foamed concrete structure management, high values of shrinkage deformations and relatively low strength characteristics.

Dispersed reinforcement with fibers is one of the promising methods to improve technological, construction and operational characteristics of cellular concretes. The use of this technology allows transmitting tensile force from composite matrix to fiber. This force transmission effect considerably reduces crack formation and opening and increases strength and crack resistance of fiber reinforced foam concrete $[2,3]$.

* Corresponding author: valeriyif.ykt@gmail.com 
In practice, synthetic fibers (polypropylene, polyethylene, nylon, ect.) are the most widely used ones. [2]. Among their advantages is almost complete resistance to aggressive alkaline medium of set cement. Synthetic fibers contribute to formation of optimum mineral frame and decrease of shrinkage deformation in foamed concrete. According to foreign researchers [3, 4], apart from the mentioned advantages the use of discontinuous fibers enables to reduce mass of a structure, improve thermal protection characteristics and fire resistance of wall structures.

The paper [5] studies foamed concrete with dispersed reinforcement with coconut fiber from the Republic of Vietnam. The fibers were milled and added to the base mix in the amount of $0.5,1,1.5$ and $2 \%$ of cement weight. The best parameters were observed for $1 \%$ of fiber content, which are $3.9 \mathrm{MPa}$ with the mean density of $830 \mathrm{~kg} / \mathrm{m}^{3}$. Use of basalt fibers for foamed concrete production is studied in [6]. The research demonstrates that formation of crystal network on the surface of basalt fibers causes considerable increase of strength parameters of fiber reinforced foamed concrete. Polyamide fibers also intensify cluster formation processes and, as a consequence, increase aggregate stability of fiber reinforced foam concrete mix [2]. At the same time, fibers can control energy and geometrical parameters of spatial bonds in water in the material structure.

Thus, discontinuous fibers shall meet the following requirements: ultimate strain of fibers shall be higher than that of concrete, relation of elastic moduli of fiber and concrete matrix shall be equal to more that 1 , ultimate tensile strength of fiber shall be higher than the same parameter for cement matrix, fibers shall be chemically stable in alkaline medium of set cement. With regard to the price/quality ratio, the so-called recycled cellulose fibers (RCF) extracted from paper waste completely meet the above requirements.

To date, both national and foreign research on the possibility of using RCF in fiber reinforced foam concrete production either lack or have the form of a review.

Due to this fact, investigation of structure formation processes in fiber reinforced foam concrete with recycled cellulose fibers, analysis of morphology and structural parameters of new formations in the contact zone between cement matrix and fibers are seen as relevant tasks in the field of obtaining foam concrete with improved properties.

The purpose of this study, therefore, is justification of the possibility to obtain fiber reinforced foam concrete of non-autoclaved hardening with improved properties based on Portland cement and the use of recycled cellulose fibers extracted from waste paper.

\section{Materials and Methods}

Technical requirements to non-autoclaved cellular concretes, including foamed concrete, are specified in the Russian Federation state standard - GOST 25485-89 "Cellular concretes. Specifications".

The subject of research is non-autoclaved structural and thermal insulating foamed concretes of the brand D600 with regard to the mean density.

Portland cement is the most often used binder for non-autoclaved concretes production according to GOST 10178 (without adding tripoli, burnt clay, trasses, glinite, silica clay and ashes) it contains no more than $6 \%$ of tricalcium aluminate $\left(\mathrm{C}_{3} \mathrm{~A}\right)$.

Silica component acceptable for foamed concrete production is sand which according to GOST 8736 contains at least $90 \%$ of $\mathrm{SiO}_{2}$, no more than $0.5 \%$ of mica and maximum $3 \%$ of silt and clay impurities.

Waste paper of brand MC-8B (newsprint) was used to extract RCF in compliance with GOST 10700-97 "Paper and cardboard waste. Specifications". This waste paper brand was selected due to its wide use and little amount of fillers and binders.

Fiber reinforced foam concrete samples were prepared by "traditional" method. Laboratory tests of the samples were performed with the use of certified equipment of the 
testing center of Yakutsk-Expert, LLC. Experimental data for qualitative and quantitative chemical analysis of the studied RCF and fiber reinforced foam concrete samples were obtained using JEOL JSM-7600F scanning electron microscope.

The experimental data and micropictures were processed in Microsoft office Excel 2007, MathCAD 2001i and ImageJ.

\section{Experimental results}

Cellulose fibers were obtained by repulping a waste paper sheet in aqueous medium. Cellulose fibers extraction from paper waste is done by combining physico-chemical and hydrodynamic processes which occur in waste paper when contacting with water. Prior to repulping waste paper was kept in aqueous medium for 12 hours. Disintegration of the sheet and its deflaking while mixing occurs under hydrodynamic and mechanical actions [7]. It shall be noted that strength of paper and cardboard fibers is much higher than that of wet sheet, therefore, mechanical actions allow splitting the sheet into fibers without their disintegration. Duration of deflaking process in a mixer was 1 hour.

Physico-mechanical parameters of fiber reinforced foam concrete samples were defined in compliance with the requirements of relevant standard technical documents $[8,9]$. RCF belong to a big group of organic fibers. The fiber looks like a hollow tube with extended coarse surface (Fig. 1). According to the results of SEM analysis the obtained RCF have the following parameters: length 5100-5300 micron, width 20-35 micron, thickness 2-5 micron, bulk density $0.08-0.1 \mathrm{~g} / \mathrm{cm}^{3}$, temperature stability up to $235^{\circ} \mathrm{C}$. RCF, like the majority of vegetable fibers, are hydrophilic, consequently, fibers are strongly glued with cement mortar.

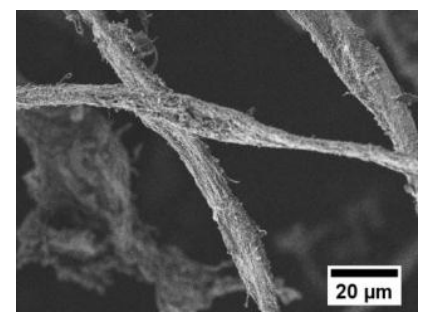

(a) $\mathrm{x} 1000$

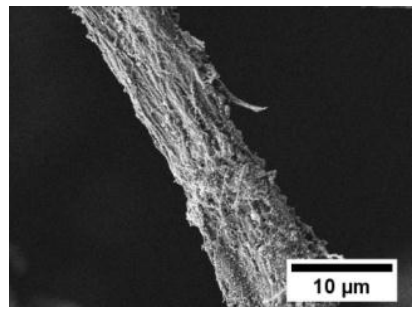

(b) $\times 3000$

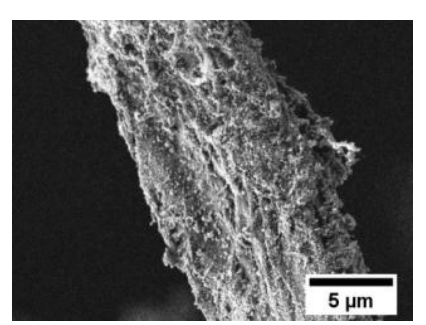

(c) $\times 5000$

Fig. 1. Microstructure of recycled cellulose fiber.

One of the key effects of using RCF in fiber reinforced foam concrete production is the ability to improve its physico-mechanical properties. The influence of RCF on strength parameters of fiber reinforced foam concrete samples was investigated in cubic specimens with the size of $100 \times 100 \times 100 \mathrm{~mm}$. The specimens have been kept at the environment temperature of $20^{\circ} \mathrm{C}$ and relative air humidity of $100 \%$ for 28 days.

Fiber reinforced foam concrete brand with regard to mean density is D600. Content of RCF was $0.1,0.2,0.3,0.4$ and $0.5 \%$ of the binder mass on a dry matter basis. Ultimate compressive strength of fiber reinforced foam concrete under compressive load of $1.9 \mathrm{MPa}$ after 28 days of natural hardening is reached with the fiber content of $0.3 \%$ wt. (compressive strength of a reference specimen is $1.4 \mathrm{MPa}$ ).

Strength decreases with further increase of fiber content. This correlation has one extremum point which, at the same time is the optimum one. Increase of strength up to the extremum value is explained by cumulative action of two factors. First, the load applied to fiber reinforced foam concrete is transmitted through cement matrix directly to fibers [2, 
$10,11]$. Second, the gain of strength is related to peculiarities of morphology of new cement formations around RCF.

Figure 2 demonstrates spectrograms of RCF and cement matrix at the age of 28 days. The following effect was determined after comparative analysis of spectrograms of RCF and products of cement hydration: higher content of calcium ions are observed in the zone of fibers location compared to the corresponding peak on the hardened cement paste spectrogram. Besides, not only the peak height increases, but its area, too. Appearance of silicon ions in RCF spectrogram shall be noted, the peak value of which is considerably lower than that of cement.

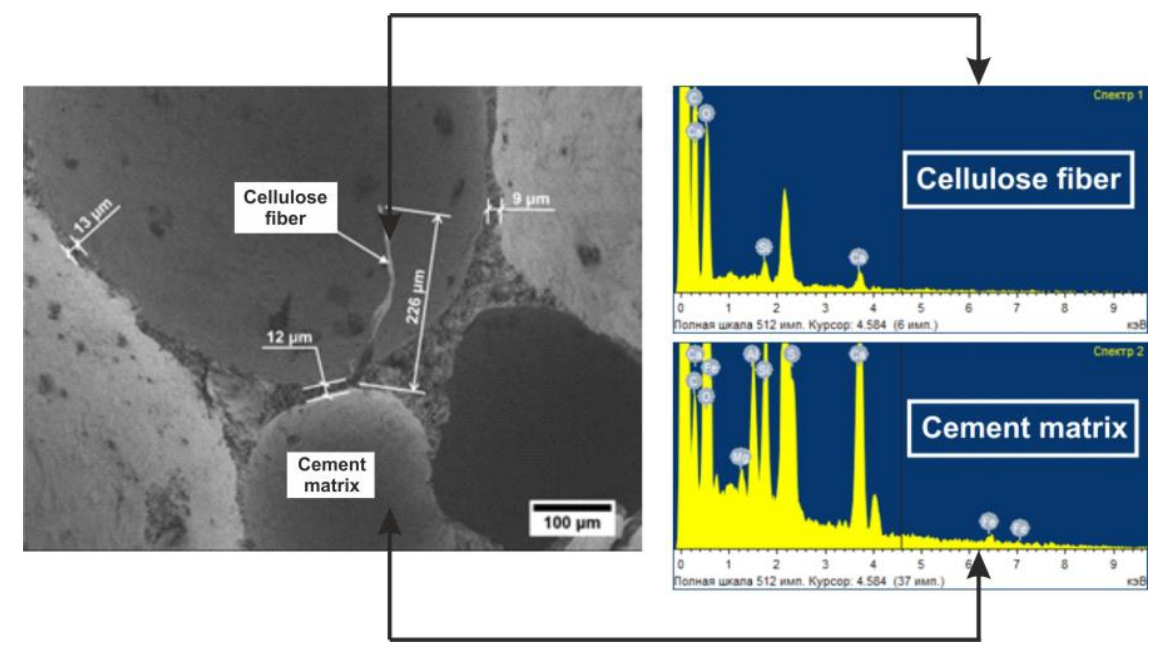

Fig. 2. Comparison of spectrograms for chemical composition of recycled cellulose fiber mixed with cement mortar and cement matrix of fiber reinforced foam concrete.

Based on the analysis of RCF and hardened cement paste spectrograms one can suggest that partial mixing of hydration products of cement clinker minerals occurred around the fiber surface layer $[12,13]$. It shall be noted that higher content of calcium ions (Table 1) might be related to formation of crystallization centers in the contact zone between RCF and cement matrix, which is followed by setting of $\mathrm{Ca}(\mathrm{OH})_{2}$ crystals $[14,15]$.

Table 1. Results of quantitative chemical analysis.

\begin{tabular}{|c|l|c|c|c|c|c|c|}
\hline \multirow{2}{*}{ No. } & \multirow{2}{*}{ Title } & \multicolumn{6}{|c|}{ Element content (reference), \% } \\
\cline { 3 - 8 } & & $\begin{array}{c}\mathrm{C} \\
\left(\mathrm{CaCO}_{3}\right)\end{array}$ & $\begin{array}{c}\mathrm{O} \\
\left(\mathrm{SiO}_{2}\right)\end{array}$ & $\begin{array}{c}\mathrm{Si} \\
\left(\mathrm{SiO}_{2}\right)\end{array}$ & $\begin{array}{c}\mathrm{Ca} \\
(\text { Wollastonite })\end{array}$ & $\begin{array}{c}\mathrm{Mg} \\
(\mathrm{MgO})\end{array}$ & $\begin{array}{c}\mathrm{Al} \\
\left(\mathrm{Al}_{2} \mathrm{O}_{3}\right)\end{array}$ \\
\hline 1 & RCF & $31-58$ & $33-43$ & $1-4$ & $6-19$ & 1 & $0.6-1$ \\
\hline 2 & Cement matrix & $5-7$ & $56-65$ & $3-8$ & $23-29$ & $0.7-1$ & $0.5-3$ \\
\hline
\end{tabular}

Microstructure of fiber reinforced foam concrete was studied for advance investigation of structure formation processes in deep layers of fiber reinforced foam concrete. Special attention was paid to studying appearance of new formations around RCF (Fig. 3).

Due to visual analysis of hydration products morphology it was found that microstructure of hardened cement paste generally consists of clusters which are composed of interlocked acicular crystals and hexagonal plates around spherical agglomerate.

First of all, new formations have high density which is proved by close packing of hydration products $[12,15]$. Besides, a thin network of microfibrillar fibers was found all over the RCF surface, their diameter being between 50 and $300 \mathrm{~nm}$ (Fig. 3, on the left). 
Presumably, microfibrils of cellulose fibers were formed because of mechanical defects during extraction from waste paper sheets. First, these fibers improve adhesion of RCF with cement matrix due to increase of fiber surface area and cause considerable gain of mechanical strength of fiber reinforced foam concrete. Second, due to ultrafine size microfibrils are sources of structure formation nuclei which increase density of interpore partition in fiber reinforced foam concrete. This leads to the increase of general strength of fiber reinforced foam concrete.

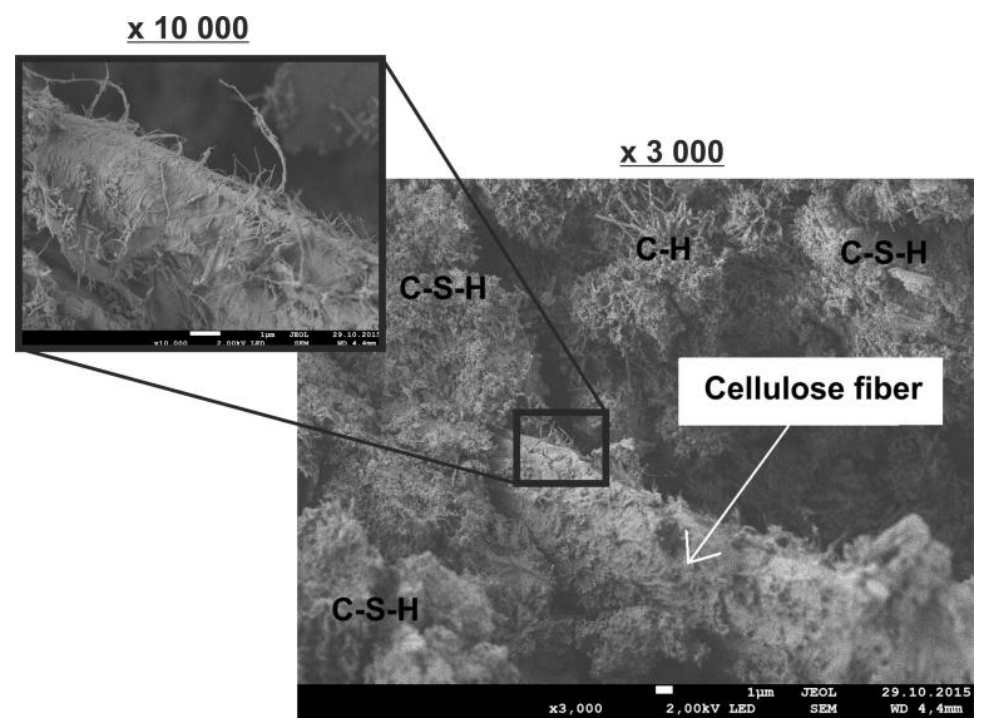

Fig. 3. Location of recycled cellulose fibers in cement matrix of fiber reinforced foam concrete. Zoom x3000 and $\times 10000$.

\section{Conclusion}

On the basis of the conducted research the conclusion can be made that the use of RCF allows increasing compressive strength of fiber reinforced foam concrete up to $35 \%$ (compared to foamed concrete without fiber addition). Besides, increase of stability of base foam concrete mixture and decrease of shrinkage deformations are observed in fiber reinforced foam concrete samples. Taking into account the fact that fibers were extracted from mere paper waste, the strength gain shall be considered quite high.

The research defines the possibility of obtaining structural thermal insulating fiber reinforced foam concrete of the brand D600 with regard to mean density and of the type $\mathrm{B} 1,5$ with regard to compressive strength with the use of RCF.

Positive effect of RCF on formation of optimum porous structure of fiber reinforced foam concrete shall be emphasized. The average diameter of macropores in samples of fiber reinforced foam concrete with RCF addition is $0.7 \mathrm{~mm}$, whereas it is $1.1 \mathrm{~mm}$ for the reference sample. Considerable reduction of the average macropore diameter is supposedly connected with redistribution of internal stresses due to the effect of dispersed reinforcement of interpore partitions and as a consequence of coalescence effect.

The extension of this research will be focused on decreasing density of fiber reinforced foam concrete with the use of recycled cellulose fibers up to $500 \mathrm{~kg} / \mathrm{m}^{3}$ with B1.5 type of concrete with regard to compressive strength. 


\section{References}

1. V.A. Perfilov, A.V. Atkina, News of higher educational institutions. Construction, 9, 26 (2010) (in Russian)

2. F.N. Rabinovich, Kompozity na osnove dispersno-armirovannyh betonov [Composites based on dispersed-reinforced concrete], 23 (ACB, Moscow, 2004) (in Russian)

3. N. Yong, Natural fibre reinforced cement composites, doctoral thesis, 127 (Victoria University of Technology, Australia, July 1995)

4. R. Khiari, Z. Marrakchi, M. N. Belgacem, E. Mauret, F. Mhenni, Composites Science and Technology, 71, 1867 (2011)

5. V.T. Nguen, Science and Technology, 9, 10 (1981)

6. N.G. Vasilovskaya, I.G. Kalugin, News of higher educational institutions. Construction, 11, 12 (2010) (in Russian)

7. D.M. Flyate, Svojstva bumagi [Paper properties], 35 (Lesnaja promyshlennost', Moscow, 1986) (in Russian)

8. GOST 10180-2012. Methods for strength determination using reference specimens (2013) (in Russian)

9. GOST 12730.1-78. Concretes. Methods of determination of density (1980) (in Russian)

10. V.I. Fedorov, A.E. Mestnikov, Industrial and civil engineering, 8, 22 (2016) (in Russian)

11. Z. Li, X. Wang, L.Wang, Composites: Part A, 37, 467 (2006)

12. Y. Chen, Evaluation of damage in cellulose fiber reinforced concrete using acoustic enission, doctoral thesis, 52 (University of Florida, 2010)

13. G.H.D. Tonoli, U.P. Rodrigues Filho, H. Savastano Jr., J. Bras, M.N. Belgacem, F.A. Rocco Lahr, Composites: Part A, 40, 2046 (2009)

14. B.J. Mohr, J.J. Biernacki, K.E. Kurtis, Cement and Concrete Research, 36, 1240 (2006)

15. R.D. Toledo Filho, K. Scrivener, G.L. England, K. Ghavami, Cement and Concrete Composites, 22, 127 (2000) 